\title{
Reducing dissemination of carbapenem-resistant Klebsiella pneumoniae
}

\author{
Matteo Bassetti ${ }^{1,2,3}$, Daniele Roberto Giacobbe ${ }^{2,3}$ \\ ${ }^{1}$ Infectious Diseases Clinic, Department of Medicine, University of Udine, Azienda Sanitaria Universitaria Integrata di Udine, Udine, Italy; \\ ${ }^{2}$ Department of Health Sciences, University of Genoa, Genoa, Italy; ${ }^{3}$ Clinica Malattie Infettive, Ospedale Policlinico San Martino IRCCS, Genoa, Italy \\ Correspondence to: Prof. Matteo Bassetti. Department of Health Sciences, University of Genoa, Genoa, Italy. Email: matteo.bassetti70@gmail.com. \\ Provenance: This is an invited article commissioned by the Section Editor Dr. Guo-Wei Tu (Department of Critical Care Medicine, Zhongshan \\ Hospital, Fudan University, Shanghai, China). \\ Comment on: Li M, Wang X, Wang J, et al. Infection-prevention and control interventions to reduce colonisation and infection of intensive care \\ unit-acquired carbapenem-resistant Klebsiella pneumoniae: a 4-year quasi-experimental before-and-after study. Antimicrob Resist Infect Control \\ 2019;8:8.
}

Submitted Aug 22, 2019. Accepted for publication Aug 30, 2019.

doi: 10.21037/atm.2019.09.11

View this article at: http://dx.doi.org/10.21037/atm.2019.09.11

Besides being resistant to several $\beta$-lactams, carbapenemresistant Klebsiella pneumoniae (CRKP) are often resistant to other widely used antimicrobials such as fluoroquinolones and cotrimoxazole, and sometimes to aminoglycosides $(1,2)$. Although possibly not the only reason, the increased risk of delayed/suboptimal treatment consequent to the multidrug-resistant phenotype of CRKP is a major contributor to the high mortality of CRKP infections (3-7). Thus, improving early diagnosis and developing novel effective antibiotics for treating CRKP infections are two important strategies for ameliorating patients' health (8-10). An essential, complementary strategy is prevention, that is, reducing the risk of development of carbapenem-resistance in carbapenem-susceptible strains by relieving selective pressure (through antimicrobial stewardship initiatives) and reducing the risk of CRKP cross-transmission (though infection-control measures) $(11,12)$. In their retrospective, before-after study, $\mathrm{Li}$ and colleagues focused on this latter factor (infection-control measures), measuring their impact in reducing the incidence rate of intensive care unit (ICU)acquired CRKP colonization/infection in a 12-bed tertiarylevel ICU in Ruijin Hospital, Shanghai, China (13).

The study was conducted over 4 consecutive periods: (I) baseline (January 2013 to June 2013); (II) implementation of infection-control measures (July 2013 to June 2014); (III) modification of infection-control measures (July 2014 to June 2015); (IV) follow-up (July 2015 to June 2016). Overall, despite some important fluctuations, the incidence rate of ICU-acquired CRKP colonization/infection decreased from 10.08 per 1,000 patient-days during the baseline period to 2.84 per 1,000 patient-days during followup, with a significant change in the slope towards reduced monthly incidence rates in linear regression models being observed between period (II) and (III), concomitant to the strengthening of infection-control measures (enhancement of external medical staff education, contact precautions of shared equipment, and enhancement terminal room disinfection in addition to measures already implemented in the previous period, i.e., active surveillance cultures, contact precautions, patient isolation in single room or cohorting, cohorting of medical care, disinfection and sterilization, contact precautions, disinfection and sterilization, and targeted bundles interventions for intravascular catheterrelated infection, ventilation associated pneumonia, and catheter-associated urinary tract) (13). The authors should be commended for their efforts in implementing these important interventions ultimately leading to a reduced incidence of newly acquired CRKP colonization/infections in the ICU. Nonetheless, from a general perspective of extrapolation and generalization of results, we think some aspects of the papers merit further discussion.

The first is that the number of cases of CRKP colonized/ 
infected patients at ICU admission increased over the study period (from 1 in the baseline period to 13 during follow-up). In this regard, it was not specified whether these patients acquired CRKP in other hospital wards before being admitted to the ICU or they acquired CRKP in other centers or in the community. In the first case (CRKP colonization/infection in other hospital wards), this may reduce the overall positive effect, since, after an initial decrease from baseline, the overall number of CRKP patients (already colonized/infected plus newly colonized/ infected) remained stable during the subsequent study periods (as possibly remained also the overall burden of CRKP-related morbidity and mortality). Against this backdrop, it would have been of interest to know as to whether targeted infection-control interventions were implemented also in other wards of the hospital and their overall effect, as well as the baseline CRKP epidemiology in other parts of the hospitals. Together with the lack of measurement of the concomitant effect of antimicrobial stewardship interventions that may have participated in positively influencing results (as acknowledged by the authors in the discussion), the lack of knowledge on what happened in other wards contributes to hamper the generalization of results. Indeed, there are important questions that readers are likely to ask themselves in the attempt to translate results to their own scenario: (I) should we focus on a single high-risk ward for strengthening infection-control measures or, in terms of overall cost-effectiveness, would it be better to consider larger interventions targeting also other selected wards or the entire hospital? (II) should we also enhance our antimicrobial stewardship efforts? (III) the intervention implemented by the authors would still work with a different baseline incidence rate of CRKP colonization/ infection as in our hospital?

Of course, even with more details it is always difficult to extrapolate local CRKP infection-control/stewardship results to other settings, and this is generally true also for other studies (14-20). In this regard, some general factors hindering generalization are the lack of control groups (almost all studies employ uncontrolled beforeafter or uncontrolled only-post designs), heterogeneity in the definitions of CRKP colonization and infection (for example, rectal swabbing is the most used method to define colonization since gastrointestinal carriage is regarded as a major reservoir of CRKP, but it is not specified if the rectum was among the multiple culture sites included in the active surveillance cultures protocol used by $\mathrm{Li}$ and colleagues), the lack of information about adherence to implemented interventions, the different follow-up periods, the different statistical models used for assessing changes in incidence trends, the inclusion of both CRKP colonization and CRKP infection in the numerator (precluding informative separate assessments), and the use of different denominators (e.g., patient-days $v s$. patient-days at risk, since the former may confound the assessment of the true incidence of newly acquired cases).

All the factors mentioned above reflect a lack of standardization both in the baseline data to be provided to readers and in measuring the impact of interventions on CRKP epidemiology. Still, undoubtedly some interventions worked. $\mathrm{Li}$ and colleagues are remarkably among the firsts to report encouraging results from China, and several other favorable experiences have been documented in other countries (13-17,19-22). Along with understanding why some other experiences were unsuccessful, increasing comparability and standardization are essential if we were to further generalize positive results, and more effectively reduce CRKP dissemination.

\section{Acknowledgments}

None.

\section{Footnete}

Conflicts of Interest: Outside the submitted work, M Bassetti has received funding for scientific advisory boards, travel and speaker honoraria from Angelini, Astellas, AstraZeneca, Basilea, Bayer, BioMérieux, Cidara, Correvio, Cubist, Menarini, Molteni, MSD, Nabriva, Paratek, Pfizer, Roche, Shionogi, Tetraphase, Thermo Fisher, and The Medicine Company. Outside the submitted work, DR Giacobbe reports personal fees from Stepstone Pharma GmbH and an unconditioned grant from MSD Italia.

Ethical Statement: The authors are accountable for all aspects of the work in ensuring that questions related to the accuracy or integrity of any part of the work are appropriately investigated and resolved.

\section{References}

1. Daikos GL, Tsaousi S, Tzouvelekis LS, et al. Carbapenemase-producing Klebsiella pneumoniae bloodstream infections: lowering mortality by antibiotic 
combination schemes and the role of carbapenems. Antimicrob Agents Chemother 2014;58:2322-8.

2. Tumbarello M, Trecarichi EM, De Rosa FG, et al. Infections caused by KPC-producing Klebsiella pneumoniae: differences in therapy and mortality in a multicentre study. J Antimicrob Chemother 2015;70:2133-43.

3. Bassetti M, Giacobbe DR, Giamarellou H, et al. Management of KPC-producing Klebsiella pneumoniae infections. Clin Microbiol Infect 2018;24:133-44.

4. Fraenkel-Wandel Y, Raveh-Brawer D, Wiener-Well Y, et al. Mortality due to blaKPC Klebsiella pneumoniae bacteraemia. J Antimicrob Chemother 2016;71:1083-7.

5. Giacobbe DR, Tumbarello M, Del Bono V, et al. Comment on: Mortality due to blaKPC Klebsiella pneumoniae bacteraemia. J Antimicrob Chemother 2016;71:1743-4.

6. Kohler PP, Volling C, Green K, et al. Carbapenem Resistance, Initial Antibiotic Therapy, and Mortality in Klebsiella pneumoniae Bacteremia: A Systematic Review and Meta-Analysis. Infect Control Hosp Epidemiol 2017;38:1319-28.

7. Sabino S, Soares S, Ramos F, et al. A Cohort Study of the Impact of Carbapenem-Resistant Enterobacteriaceae Infections on Mortality of Patients Presenting with Sepsis. mSphere 2019. doi: 10.1128/mSphere.00052-19.

8. Avery LM, Nicolau DP. Investigational drugs for the treatment of infections caused by multidrug-resistant Gram-negative bacteria. Expert Opin Investig Drugs 2018;27:325-38.

9. Bassetti M, Peghin M, Vena A, et al. Treatment of Infections Due to MDR Gram-Negative Bacteria. Front Med (Lausanne) 2019;6:74.

10. Giacobbe DR, Mikulska M, Viscoli C. Recent advances in the pharmacological management of infections due to multidrug-resistant Gram-negative bacteria. Expert Rev Clin Pharmacol 2018;11:1219-36.

11. Bassetti M, Giacobbe DR, Vena A, et al. Challenges and research priorities to progress the impact of antimicrobial stewardship. Drugs Context 2019;8:212600.

12. Tacconelli E, Cataldo MA, Dancer SJ, et al. ESCMID guidelines for the management of the infection control measures to reduce transmission of multidrug-resistant Gram-negative bacteria in hospitalized patients. Clin Microbiol Infect 2014;20 Suppl 1:1-55.

13. Li M, Wang X, Wang J, et al. Infection-prevention and control interventions to reduce colonisation and infection of intensive care unit-acquired carbapenem-resistant
Klebsiella pneumoniae: a 4-year quasi-experimental before-and-after study. Antimicrob Resist Infect Control 2019;8:8.

14. Abdallah M, Olafisoye O, Cortes C, et al. Rise and fall of KPC-producing Klebsiella pneumoniae in New York City. J Antimicrob Chemother 2016;71:2945-8.

15. Cohen MJ, Block C, Levin PD, et al. Institutional control measures to curtail the epidemic spread of carbapenemresistant Klebsiella pneumoniae: a 4-year perspective. Infect Control Hosp Epidemiol 2011;32:673-8.

16. Frattari A, Savini V, Polilli E, et al. Control of Gramnegative multi-drug resistant microorganisms in an Italian ICU: Rapid decline as a result of a multifaceted intervention, including conservative use of antibiotics. Int J Infect Dis 2019;84:153-62.

17. Giacobbe DR, Del Bono V, Mikulska M, et al. Impact of a mixed educational and semi-restrictive antimicrobial stewardship project in a large teaching hospital in Northern Italy. Infection 2017;45:849-56.

18. Kousouli E, Zarkotou O, Politi L, et al. Infection control interventions affected by resource shortages: impact on the incidence of bacteremias caused by carbapenem-resistant pathogens. Eur J Clin Microbiol Infect Dis 2018;37:43-50.

19. Viale P, Tumietto F, Giannella M, et al. Impact of a hospital-wide multifaceted programme for reducing carbapenem-resistant Enterobacteriaceae infections in a large teaching hospital in northern Italy. Clin Microbiol Infect 2015;21:242-7.

20. Xu YL, Hu LM, Xie ZZ, et al. [Impact of antimicrobial stewardship program on antimicrobial usage and detection rate of multidrug-resistant gram-negative bacteria]. Zhonghua Er Ke Za Zhi 2019;57:553-8.

21. Ben-David D, Masarwa S, Adler A, et al. A national intervention to prevent the spread of carbapenem-resistant Enterobacteriaceae in Israeli post-acute care hospitals. Infect Control Hosp Epidemiol 2014;35:802-9.

22. Hayden MK, Lin MY, Lolans K, et al. Prevention of colonization and infection by Klebsiella pneumoniae carbapenemase-producing enterobacteriaceae in long-term acute-care hospitals. Clin Infect Dis 2015;60:1153-61.

Cite this article as: Bassetti M, Giacobbe DR. Reducing dissemination of carbapenem-resistant Klebsiella pneumoniae. Ann Transl Med 2019;7(Suppl 8):S365. doi: 10.21037/ atm.2019.09.11 